\title{
Fast Realistic Multi-Action Recognition using Mined Dense Spatio-temporal Features
}

\author{
Andrew Gilbert, John Illingworth and Richard Bowden \\ CVSSP, University of Surrey, Guildford, Surrey GU2 7XH United Kingdom \\ a.gilbertesurrey.ac.uk
}

\begin{abstract}
Within the field of action recognition, features and descriptors are often engineered to be sparse and invariant to transformation. While sparsity makes the problem tractable, it is not necessarily optimal in terms of class separability and classification. This paper proposes a novel approach that uses very dense corner features that are spatially and temporally grouped in a hierarchical process to produce an overcomplete compound feature set. Frequently reoccurring patterns of features are then found through data mining, designed for use with large data sets. The novel use of the hierarchical classifier allows real time operation while the approach is demonstrated to handle camera motion, scale, human appearance variations, occlusions and background clutter. The performance of classification, outperforms other state-of-the-art action recognition algorithms on the three datasets; KTH, multi-KTH, and realworld movie sequences containing broad actions. Multiple action localisation is performed, though no groundtruth localisation data is required, using only weak supervision of class labels for each training sequence. The realworld movie dataset contain complex realistic actions from movies, the approach outperforms the published accuracy on this dataset and also achieves real time performance.
\end{abstract}

\section{Introduction}

Action recognition shares many of the problems associated with object recognition from static images such as the ability to deal with intra class variation, occlusion, scale and background clutter. In the context of object recognition, these problems are generally solved using the bag of words or feature based approaches [26] combined with a state of the art machine learning technique such as a Boosting [25] or Support Vector Machines [21]. However, it has not been proven that this can be directly transferred into the temporal domain, for action recognition, without compromise. Given the quantity of images in a video sequence, the features used are often engineered to be sparse and fire rarely to reduce overheads. These sparse features are assumed to be the most descriptive for the learnt actions, however, it is possible that the sparsity disregards important discriminatory information.

Building on the success of data mining within other fields $[20,6,2]$, we propose to use data mining within the action recognition field to allow a multi stage classifier to be learnt from a large set of simple features. Initially a very localised neighbourhood grouping is used to form compound grouping of features. The neighbourhood is hierarchically increased until the final stage uses only the relative position of groupings to provide scale invariance. The novel hierarchical approach allows for real time operation and out performs other state of the art approaches on the KTH dataset [21]. Finally we present results on two more challenging sequences. Multi-KTH [24] to demonstrate its performance at classifying and localising multiple actions in noisy cluttered scenes containing camera motion and the extremely challenging data of [12] which contains segments extracted from a variety of movies.

\section{Related Work}

Much of the early work in action recognition was tested on relatively simple single person uniform background sequences $[21,7]$. To address this, more natural and diverse video settings are being developed. Laptev et al [13] developed a motion model using optical flow [3] [16] to recognise smoking and drinking actions within a feature length film. Uemura et al [24] used a motion model based on optical flow combined with SIFT feature correlation in order to accurately classify multiple actions on a moving camera sequence. Laptev et al [12] used space time features within a spatio-temporal bag of words model on a large selection of movie film clip actions. While within object recognition, success has been shown by bag of words approaches [20, 14, 23]. The bag of words approach has also been extended to the temporal domain $[21,4,18,12]$. However to scale to the temporal domain, the occurrence 
of the complex feature must be kept sparse. Scovanner et al [22] extended the 2D Harris detector and 2D SIFT descriptor [15] into three dimensions, causing the feature to fire far less often. Similarly, Willems et al [27] extended the 2D hessian detector and SURF descriptor to the spatiotemporal domain.

Quack et al [20] applied Association rule data mining to object recognition by mining spatially grouped SIFT descriptors. We have previously applied a similar approach to mining actions from video. This paper extends previous work published by Gilbert et al [6]. The previous work focused on single person classification on the simple KTH dataset. This paper extends the technique to multi person detection and localisation, and introduces a hierarchical application of the mining technique which provides a significant performance increase. Furthermore, the hierarchy provides both speed and scalability which allows the real time application of the approach to detecting complex actions in movies.

The main contributions of this paper are the use of a hierarchical classifier to provide a fast accurate multi-action classifier using data mining and the localisation and recognition of simultaneous multiple actions. Our approach out performs all previously published work on the three datasets that we test upon. The simple features used are explained in section 3, and how they are grouped in the hierarchical classifier in section 4 . The training and recognition of the classifier is shown in section 5, results and a conclusion are presented in sections 6 and 7 .

\section{Features}

Space-time features have shown good performance for action recognition $[21,4]$. They can provide a compact representation of interest points, with the ability to be invariant to some image transformations. While many are designed to be sparse in occurrence $[21,4,12]$, we use dense simple 2D harris corners [8]. In [6] we maintained a relatively simple neighbourhood grouping. Here we increase the detail of the neighbourhood through the hierarchical classifier, this improves the speed and accuracy of the approach dramatically. The features are detected in $(x, y),(x, t)$ and $(y, t)$ channels in the sequences. This provides information on spatial and temporal image changes but is a far denser detection rate than 3D harris corners [11, 12] and encodes both spatial and spatio-temporal aspects of the data. The overcomplete set of features are then reduced through the levels of mining. The interest points are extracted at multiple levels of spatial scales $\left(\sigma_{i}\right)$ with $\sigma_{i}=3 * 2^{i-1}$, where $i=1, \ldots 5$. This covers scales of corner interest points from $3 \times 3$ up to $48 \times 48$ pixels in size. Figure 1 shows the example corner detections on two frames. Figure 1(b) shows the large amount of corner detections on the multi-KTH dataset [24] where there are around 1500 corners detected per frame.

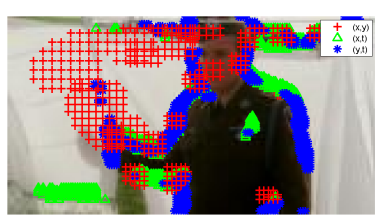

(a)

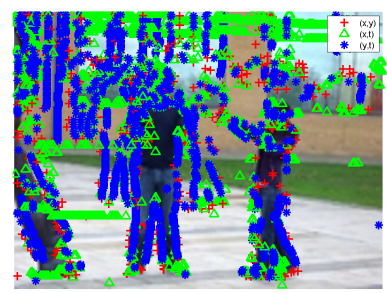

(b)
Figure 1. Corner Detection on two Frames, (a) An example of HugPerson from Laptev Movie Dataset [12], (b) Boxing and Handclapping examples from Multi-KTH Dataset [24]

To characterize the motion of the interest points, the dominant orientation of the corners is also recorded. This is quantised into $k$ discrete orientations. In our experiments $k=8$, therefore the orientation is quantised into $\frac{1}{4} \pi$ bins aligned with the points of a compass. For each interest point detected, it is represented by a 3 digit code [Scale, Channel, Orientation]: The scale at which the interest point was detected $S$ cale $=\{1, \ldots, 5\}$. The channel the interest point was detected in Channel $=1, \ldots, 3$ with $1=(x, y), 2=(x, t)$ and $3=(y, t)$. Finally the dominant orientation of the corner quantised into $\frac{1}{4} \pi$ bins, so Orientation $=\{1, \ldots, 8\}$.

\section{Neighbourhood Grouping}

Given a set of encoded corners, their relationships to other corners are used to build stronger compound features. A hierarchical neighbourhood grouping is proposed, that allows initial stages to identify short localised compound features. Subsequent levels then group these compound features into increasingly higher level spatio-temporal features. The final stage encoding the relative positions of compound features. This hierarchical process is designed to speed up the overall classification and improve accuracy in a similar way to that of a cascaded classifier [5].

\subsection{Hierarchical Grouping}

The dense corner features are hierarchically grouped into increasingly higher level compound features. This has the advantage of being fast to compute and able to produce more complex features to pass to the successive grouping stages. A regular grid is used to encode spatial information about the local neighbourhood of an interest point. Each neighbourhood is divided into $3 \times 3 \times 3$ cuboids with each cuboid $\omega$ pixels wide and high. Figure 2 shows an illustration of the process. The grid extends in space and time in order to use the important temporal information concurrently. The cuboids within the grid are labelled seqeuncetially from 0 to 26 and the grid is centerd over each interest point in turn. Any other interest points that occur within the grid 
neighbourhood have their ID prefixed with the label of the cuboid as shown in Figure 2. After all neighbouring interest

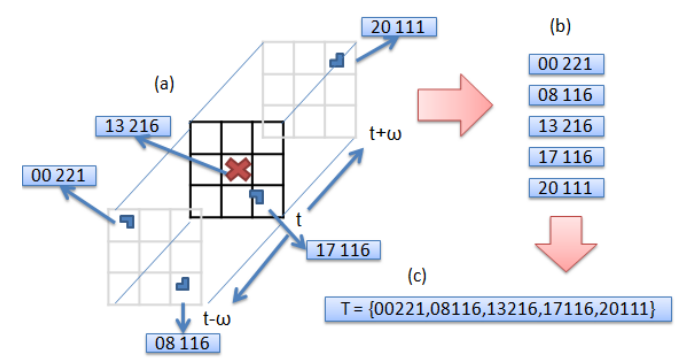

Figure 2. (a) Shows the grid design centerd on the interest point marked as a cross, with five local features shown as corners. (b) shows the spatial and temporal encoding applied to each local feature. (c) Concatenating the local features into a transaction vector $T$ for this interest point.

points have been prefix with their specific cuboid label they are concatenated into a $1 \mathrm{D}$ vector. This vector of neighbouring interest points is called a transaction vector, $T$, where each of the neighbouring interest points are termed items. We are especially interested in learning discriminative configurations, which appear frequently in the specific action sequence, and rarely on other actions or the background. Therefore, for each action class the transaction vectors are appeneded with a specific action label. For example, the complete transaction for the interest point in Figure 2 is $\{01221,08116,13216,18116,21111$, action $\}$. In order to identify the frequently reoccurring patterns within the transaction vectors, association rule data mining is used. All the transaction vectors from all action training sequences are combined into a single database and fed into a data mining algorithm called APriori [1]. The APriori association rule algorithm is applied to the transaction database, this computationally efficient approach, finds the subsets of the transaction vectors which are frequently occurring in other transaction vectors of the same action class. The resultant subsets are called frequently reoccurring itemsets. The resulting frequent configurations are then used to group the corner features over a larger grid in the next hierarchical stage. For a greater explanatation of the apriori data mining approach see the previous work by Gilbert et al [6].

\section{Action recognition}

Equipped with the approach described previously, it is now possible to learn distinctive high level features to describe an action sequence from a very large set of simple 2D corners.

\subsection{Training}

For action recognition, the simple corner features described in section 3 are densely detected and each feature is encoded with its three digit value.

For the current work, a two stage hierarchical grouping process is used, the initial stage uses a very localised neighbourhood where the width and height of each neighbourhood tile, $\omega$ is 1 pixel, further levels of the hierarchy do not give significant improvement on this data as will be discussed in section 6.4. This first stage of grouping, produces around 7 million transaction vectors. For most clustering methods this would be difficult to use, however with the association rule mining it takes around 1 minute to compute the frequent configurations $M^{1}$, of the corners.

The second and final stage of grouping uses a slightly different grid design to enable scale invariance but the method is as before. The original $2 \mathrm{D}$ corners are now matched to the frequent configurations using a look-up to the learnt compound features from the first iteration stage, $M^{1}$ using a global classifier based on voting.

\subsection{Global Classifier}

The global classifier exhaustively compares a specific frequent configuration $M_{\alpha}^{l}$, for action $\alpha$ and grouping iteration $l$, with the original $2 \mathrm{D}$ corner combinations in the query transaction set $T$ grouped within their local neighbourhoods. It works as a voting scheme by accumulating the occurrences of the mined compound features within the query transaction vector. This provides a confidence for each set of items within the query transaction vector.

$$
\operatorname{Conf} f_{\alpha}=\operatorname{Norm} * \sum_{i=0}^{\text {sizeof }(T)} \sum_{j=0}^{\text {sizeof }\left(M_{\alpha}^{l}\right)} m\left(T_{i}, M_{\alpha}^{l}\right)
$$

Where sizeof $(T)$ is the number of subsets within $T, N$ orm is the normalisation factor, shown in Equation 2

$$
\text { Norm }=\frac{1}{\operatorname{sizeof}(T) * \operatorname{sizeof}\left(M_{\alpha}^{l}\right)}
$$

and $m\left(T_{i}, M_{\alpha}^{l-1}\right)$ describes if a mined compound feature set is present in the transaction vector as in Equation 3

$$
m\left(T_{i}, M_{\alpha j}^{l}\right)=\left\{\begin{array}{cl}
\operatorname{Conf}\left(T_{i} \Rightarrow \alpha\right) & T_{i} \in M_{\alpha}^{l} \\
0 & \text { otherwise }
\end{array}\right.
$$

\subsection{Scale Invariant grouping}

If a transaction vector, $T_{i}$, has a confidence greater than zero, the corresponding mined vector, $M_{\alpha j}^{l}$, is saved as a compound feature. Repeated over all features, this will form compound features for the sequence. Each compound feature has a grid centerd over it, however, to allow for scale invariance, only the relative spatial displacement in terms of angle rather than spatial distance is used. This means the grid is adjusted to be $2 \times 2 \times 3$. Divided into 12 equal 3D 
boxes in the $x, y, t$ domain where $x, y$ radiate from the centre of the neighbourhood out to infinity, and $t$ extends to successive and preceding frames based on $\omega$. The grid design for a single frame is shown in Figure 3. The resulting

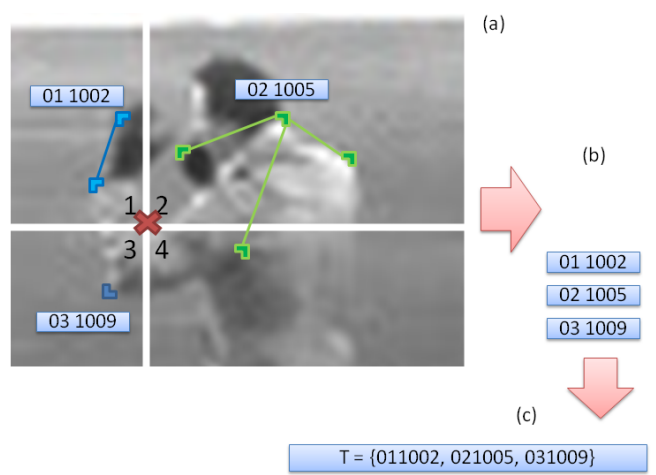

Figure 3. Note for illustration a single frame grounding is shown. (a) There are three compound features found on this frame with the IDs 1002,1005 , and 1009, these have been prefixed by there grid location in (b). (c) Shows the concatenation of the compound features into a transaction vector for this interest point.

transactions of the compound features are collated. This stage of training produces around 12 million transactions, with each transaction containing over 1000 items (encoded compound features). Association rule mining is applied to the transactions, to identify the reoccurring compound patterns. This mining is computed in around 30 minutes which is impressive. The resultant frequent configurations $M_{\alpha}^{2}$, contains around 500 association rules.

\subsection{Recognition}

The resulting Frequent Configurations $\mathbf{M}_{\alpha}^{2}$ represents the frequent and distinctive itemsets of the training action sequences. To classify an unknown test sequence, the sequence is analysed and the spatio-temporal compound 2D features are detected on a frame by frame basis to form transaction vectors as with the training. These query transaction vectors are then voted on using the global classifier presented previously in section 5.2. This is repeated over the complete test sequence to find the action likelihood of the sequence. A correct match will occur often in equation 3 as the mining will select frequently reoccurring items that are distinct compared to other actions. Each video sequence is then classified as the action, $\alpha$, for which the votes are maximised.

\subsection{Localisation}

Each vote from the global classifier on the query transaction vector gives a likelihood of an action. This can be used with a sliding window in order to localise the action. The likelihood is maximised over a window applied at multiple scales. The windows centre position is moved over the complete image, and an integral image is used for efficiency.

\section{Experimental Results}

Three sets of data were used to test the approach proposed within this paper. The well known and popular KTH dataset [21] the more challenging, multi-KTH [24], and a real-world example dataset of movie actions [12]. The KTH dataset contains 6 different actions; boxing, hand-waving, hand-clapping, jogging, running and walking. The state of the art accuracy values on the KTH dataset is around $86 \%$ to $95 \%$, therefore multi-KTH was proposed to provide more challenging tests. It consists of a single 753 frame long sequence where multiple people perform the KTH actions simultaneously. To increase difficulty, there are large scale changes and camera motion and a non uniform background. The Real-World Movie dataset of Laptev [12] consists of 8 actions; AnswerPhone, GetOutCar, Handshake, HugPerson, Kiss, SitDown, SitUp, StandUp.

\subsection{Performance Measures}

On the KTH dataset, the data for training and testing can be partitioned in two ways. The partition originally proposed by Schüldt [21] is focused on to allow direct comparison of results. For each action, 8 different people's sequences are used for training and testing. Many authors ignore the partitioning and instead opt for a far easier leaveone-out cross validation. While this is a far similar test, we also report results to allow comparison. For the multi$\mathrm{KTH}$, accuracy of the localisation is used as the measure of performance. The localisation is deemed correct if the dominant pixel label within the groundtruth bounding box matches the groundtruth. Visual examples of the localisation are shown in Figure 5. The clean test and training partitions used by Laptev [12] were used for the Movie Dataset to allow direct comparison to their published results.

\subsection{Action Classification}

The classification of the actions within the KTH dataset is performed to allow a comparison of results with other of the state of art approaches. Figure 4 shows the confusion matrix for the KTH data. There is most confusion occurring between jogging and running and between handclapping and handwaving.

Table 1 shows our proposed technique has a higher classification accuracy than all other state of the art methods. Including Subseq Boost Nowozin: The boosted SVM classifier by Nowozin [19], and Data Mined The simple single level mining approach by Gilbert [6]

Results within Table 2 use the simpler leave-one-out approach. We also report results using this data with an average performance of $96.7 \%$. Again, this outperforms all 


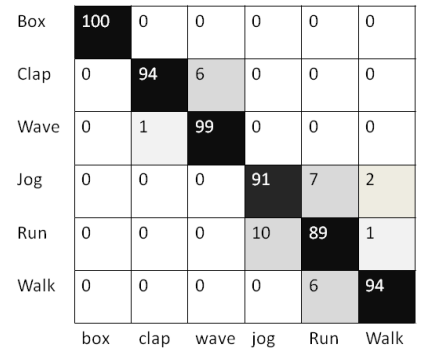

Figure 4. Human action recognition results, confusion matrix for KTH dataset

Table 1. Comparison of Average precision compared to other techniques on KTH action recognition Dataset using training/test partition proposed by [21]

\begin{tabular}{|c|c|}
\hline $\begin{array}{c}\text { Method } \\
\text { Schüldt training/test partitions }\end{array}$ & $\begin{array}{c}\text { Average } \\
\text { Precision }\end{array}$ \\
\hline \hline Gilbert et al [6] Data Mined & $89.9 \%$ \\
Nowozin et al [19] Subseq Boost SVM & $87.04 \%$ \\
Schüldt et al [21] SVM Split & $71.71 \%$ \\
Ke et al [9] Vol Boost & $62.97 \%$ \\
\hline \hline Our Approach & $94.50 \%$ \\
\hline
\end{tabular}

Table 2. Comparison of Average precision compared to other techniques on KTH action recognition Dataset using leave-one-out cross validation.

\begin{tabular}{|c|c|}
\hline Method & $\begin{array}{c}\text { Average } \\
\text { Precision }\end{array}$ \\
\hline \hline leave-one-out test/train & $95 \%$ \\
Kim et al [10] CCA & $93.7 \%$ \\
Uemura et al [24] Motion Comp Feats & $86.60 \%$ \\
Wong and Cipolla [28] Subspace SVM & $81.50 \%$ \\
Niebles et al [17] pLSA model & $81.20 \%$ \\
Dollar et al [4] Spat-Temp & $96.7 \%$ \\
\hline \hline Our Approach
\end{tabular}

other approaches and gives an indication of the complexity of leave-one-out cross validation versus the training, testing split of [21].

To test on the more challenging multi-KTH dataset, the KTH training setup was used and the localised detection results are displayed in Table 3 using the localisation accuracy metric described in section 6.1 . The previously published results from Uemura [24] are also shown to provide a benchmark. Our approach has superior performance and has a lower computation costs as is shown in section 6.3. Visual localisation results are shown in Figure 5. In Addition, the approach by Uemura required groundtruthed positions during training to be able to localise actions while our approach required no spatial groundtruth data.
Table 3. Comparison of Average precision of actions compared to other techniques on multi-KTH action recognition Dataset.

\begin{tabular}{|c||c|c|c|c|c||c|}
\hline & Clap & Wave & Box & Jog & Walk & Ave \\
\hline$[24]$ & $76 \%$ & $81 \%$ & $58 \%$ & $51 \%$ & $61 \%$ & $65.4 \%$ \\
Ours & $75 \%$ & $84 \%$ & $76 \%$ & $50 \%$ & $59 \%$ & $68.8 \%$ \\
\hline
\end{tabular}

(a)

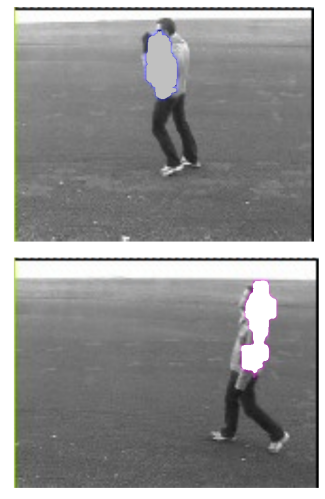

(b) (c)

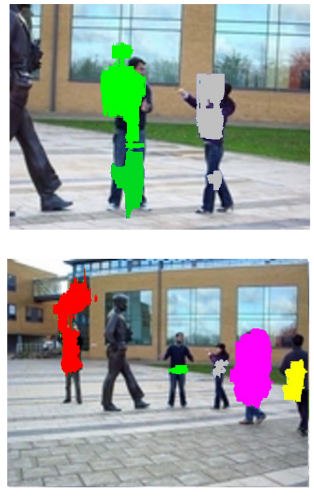

(d)
Figure 5. Corner Detection on fours Frames, (a) KTH Boxing Sequence, (b) KTH Running Seqeunce (c) Multi-KTH Boxing and Handclapping, (d) Multi-KTH all actions

Comparing the results with the original KTH data, the average accuracy is reduced by just over $25 \%$. This demonstrates the more challenging nature of the multi-KTH data.

\subsection{Computation of KTH dataset}

One of the advantages of using a data mining technique such as association rules, is the speed of learning patterns within the data. Within this paper the classification and training stages have a low computational cost. This is due to the use of the multi stage hierarchical grouping process. The use of a localised first stage to build compound features, allows high speed matching in the second and main stages where the neighbourhoods are far larger but the number of features far less. To illustrate this, table 4 shows the average frame rate for the stages of the approach. The table shows that the speed of the approach is fast throughout the entire process despite being an unoptimised $\mathrm{C}++$ implementation running on a standard single core desktop PC. In addition the number of features is greatly reduced by the second iteration, this is the main reason for the speed being maintained despite an additional level of complexitly. Overal the number of features detected has an effect on the overal speed as can be seen by the differeing frame rate between the KTH and the Multi-KTh and Hollywood datasets. However by further increasing the levels of classfier for the more complex seqeunces could maintain real time operatation despite 
Table 4. A break down of the average frame per second and features per frame of the successive stages of the classifer

\begin{tabular}{|c|c|c|}
\hline Stage & $\begin{array}{c}\text { Frames per } \\
\text { second }\end{array}$ & $\begin{array}{c}\text { Ave features } \\
\text { per frame }\end{array}$ \\
\hline Training Iter 1 & 35fps & 1500 \\
Mining Iter 1 & 640fps & \\
Training Iter 2 & 28fps & 300 \\
Mining Iter 2 & $21 \mathrm{fps}$ & \\
\hline \hline Test KTH & 24fps & 400 \\
Test multi-KTH & $4 \mathrm{fps}$ & 1500 \\
Test Hollywood & 6fps & 1300 \\
\hline
\end{tabular}

an increasing number of detected features.

\subsection{Real-World movie actions}

Training is performed with the clean, manual dataset of 219 videos. The performance is evaluated using the clean test dataset of 217 videos. This is the same as reported by Laptev in [12] to allow for a direct comparison of results. Table 5 presents the average accuracy for the 8 actions These result show a $15 \%$ improvement over the previously

Table 5. Average precision for each action class of the real-world Movie test set. We compare our approach with the previously published results.

\begin{tabular}{|c||c|c|c|}
\hline \multirow{2}{*}{ Action } & $\begin{array}{c}{[12]} \\
\text { Acc }\end{array}$ & $\begin{array}{c}\text { 2 Stage } \\
\text { Classifier }\end{array}$ & $\begin{array}{c}\text { 3 Stage } \\
\text { Classifier }\end{array}$ \\
\hline AnswerPhone & $32.1 \%$ & $25.7 \%$ & $41 \%$ \\
GetOutCar & $41.5 \%$ & $38.5 \%$ & - \\
HandShake & $32.3 \%$ & $45.6 \%$ & $52 \%$ \\
HugPerson & $40.6 \%$ & $42.8 \%$ & - \\
Kiss & $53.3 \%$ & $72.5 \%$ & - \\
SitDown & $38.6 \%$ & $84.6 \%$ & $9 \%$ \\
SitUp & $18.2 \%$ & $29.4 \%$ & $31 \%$ \\
StandUp & $50.5 \%$ & $41.6 \%$ & $24 \%$ \\
\hline \hline Average & 38 & $53.5 \%$ & $31.4 \%$ \\
\hline
\end{tabular}

published work using the 2 stage classifier, on what is a very difficult but real-world dataset, containing varying camera angles, illumination and people. An illustration of the difficulty is shown in Figure 6 which shows key frames from two sequences within the SitUp class. Using the 3 stage classifier there are promising results in many of the action classes. With three actions showing improved results on the 2 stage classifier. However as the complexity of the compound features increases with additional levels of mining, the number of features that fire reduces. At stage three this becomes a problem and dashes (-) show actions that could not be learnt at stage three due to insufficient data in train-
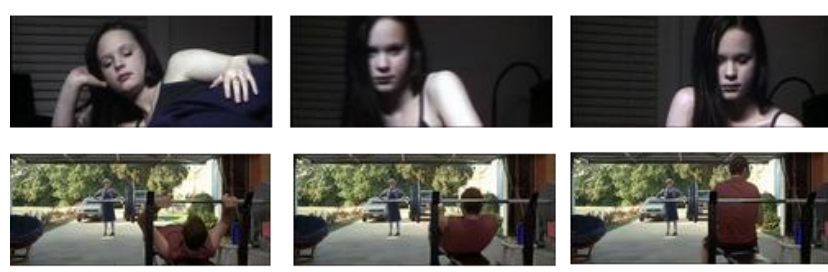

Figure 6. Key Frames from two SitUp Sequences

ing. This is also evident in reduction of the SitDown action at stage three.

Further analysis of why the approach works was performed, the key point to the method is the combination of spatially grouped compound features. Therefore a test was performed on the movie dataset where the minimum number of items within a mined transaction vector was increased. Table 6 shows the average accuracy and also frame rate as the minimum number of items (compound corners) is increased. The increase in accuracy as the minimum item

Table 6. Average precision of Movie dataset, increasing the minimum number of items within the Transaction vector.

\begin{tabular}{|c||c|c|c|c|c|}
\hline $\begin{array}{c}\text { Min No. } \\
\text { of Items }\end{array}$ & 2 & 3 & 4 & 5 & 6 \\
\hline Accuracy & $0 \%$ & $9.6 \%$ & $21 \%$ & $52 \%$ & $54 \%$ \\
Min fps & 0.0001 & 0.002 & 0.2 & 3.5 & 4.5 \\
Max fps & 0.001 & 0.1 & 2 & 28 & 28.5 \\
\hline
\end{tabular}

size increases, indicates that the greater the size of the compound feature the more important it is within the classifier. This is intuitive as the more complex compound features are likely to contain greater discriminative detail for improved inter class disambiguation. The other advantage of the hierarchical approach is that as the minimum number of items is increased, so does the the frame rate. This is due to the reduced total number of transaction vectors within the classifier.

\section{Conclusion}

This paper has presented a method to recognise and localise actions performed by different humans in real time. This is achieved through the use of compound 2D corner features. The simple corners detected form an overcomplete set of features are encoded with respect to their spatial and temporal neighbourhood. Compound corner groups are formed using a multi-stage grouping. Data mining is used to reduce the overcomplete set to form short feature sets that frequently occur in the training data. These compound features are used to produce localised likelihoods of actions in three sequences; the KTH dataset, the multi-KTH dataset where multiple objects and actions occur simultane- 
ously and a real-world Movie dataset. Performance is better than previously achieved results for all datasets, while real time operation is also possible on the datasets due to the implementation of the hierarchical neighbourhood grouping. While the localisation of multiple actions occurs with no object segmentation supplied during training.

\section{References}

[1] R. Agrawal and R. Srikant. "Fast Algorithms for Mining Association Rules in Large Databases". In VLDB'94, Proceedings of 20th International Conference on Very Large Data Bases, pages 487-499, 1994.

[2] O. Chum, J. Philbin, and A. Zisserman. Near Duplicate Image Detection: min-Hash and tf-idf Weighting. In Proc. of BMVA British Machine Vision Conference (BMVC'08), 2008.

[3] N. Dalal, B. Triggs, and C. Schmid. "Human Detection using Oriented Histograms of Flow and Apperance". In Proc. of European Conference on Computer Vision (ECCV'06), II:428-441, 2006.

[4] P. Dollar, V. Rabaud, G. Cottrell, and S. Belongie. "Behavior Recognition via Sparse Spatio-temporal Features". ICCCN '05: Proceedings of the 14th International Conference on Computer Communications and Networks, pages 65-72, 2005.

[5] Y. Freund and R. E. Schapire. "Experiments with a New Boosting Algorithm". Proc. The 13th Conf on MAchine Learning, pages 148-156, 1996.

[6] A. Gilbert, J. Illingworth, and R. Bowden. "Scale Invariant Action Recognition Using Compound Features Mined from Dense Spatio-Temporal Corners". In Proc. of European Conference on Computer Vision (ECCV'08), I:222233, 2008.

[7] L. Gorelick, M. Blank, E. Shechtman, M. Irani, and R. Basri. "Actions as Space-Time Shapes". In IEEE Trans PAMI, 29(12):2247-2253, December 2007.

[8] C. Harris and M. Stphens. "A Combined Corner and Edge Detector". In Proc. of Alvey Vision Conference, pages 189192, 1988.

[9] Y. Ke, R. Sukthankar, and M. Hebert. "Efficient Visual Event Detection using Volumetric Features". In Proc. of IEEE International Conference on Computer Vision (ICCV'05), 2005.

[10] T. Kim, S. Wong, and R. Cipolla. "Tensor Canonical Correlation Analysis for Action Classification". In Proc. of IEEE International Conference on Computer Vision and Pattern Recognition (CVPR'07), pages 1-8, 2007.

[11] I. Laptev and T. Lindeberg. "Space-time Interest Points". In Proc. of IEEE International Conference on Computer Vision (ICCV'03), pages 432-439, 2003.

[12] I. Laptev, M. Marszalek, C. Schmid, and B. Rozenfeld. "learning Realistic Human Actions from Movies". In Proc. of IEEE International Conference on Computer Vision and Pattern Recognition (CVPR'08), pages 1-8, 2008.

[13] I. Laptev and Pérez. "Retrieving Actions in Movies". In Proc. of IEEE International Conference on Computer Vision (ICCV'07), 2007.
[14] S. Lazebnik, C. Schmid, and J. Ponce. "Semi-Local Affine Parts for Object Recognition". In Proc. of BMVA BMVC'04, II:959-968, 2004.

[15] D. Lowe. "Distinctive Image Features from Scale-Invariant Keypoints". In International Journal of Computer Vision, 20:91-110, 2003.

[16] B. Lucas and T. Kanade. "An Iterative Image Registration Technique with an Application to Stereo Vision". In Proc. of 7th International Joint Conference on Artificial Intelligence (IJCAI), pages 674-679, 1998.

[17] J. Niebles, H. Wang, and L. Fei-Fei. "Unsupervised Learning of Human Action Categories using Spatial-Temporal Words". In Proc. of BMVA British Machine Vision Conference (BMVC'06), III:1249-1259, 2006.

[18] J. C. Niebles and L. Fei-Fei. "A Hierarchical Model of Shape and Appearance for Human Action Classification". In Proc. of IEEE International Conference on Computer Vision and Pattern Recognition (CVPR'07), 2007.

[19] S. Nowozin, G. Bakir, and K. Tsuda. "Discriminative Subsequence Mining for Action Classification". In Proc. of IEEE International Conference on Computer Vision (ICCV'07), pages 1919-1923, 2007.

[20] T. Quack, V. Ferrari, B. Leibe, and L. Gool. "Efficient Mining of Frequent and Distinctive Feature Configurations". In Proc. of IEEE International Conference on Computer Vision (ICCV'07), 2007.

[21] C. Schuldt, I. Laptev, and B. Caputo. Recognizing Human Actions: a Local SVM Approach. In Proc. of International Conference on Pattern Recognition (ICPR'04), III:32-36, 2004.

[22] P. Scovanner, S. Ali, and M. Shah. "A 3-dimensional Sift Descriptor and its Application to Action Recognition". In In Proc. of MULTIMEDIA '07, pages 357-360, 2007.

[23] J. Sivic and A. Zisserman. "Video Data Mining using Configurations of Viewpoint Invariant Regions". In Proc. of IEEE International Conference on Computer Vision and Pattern Recognition (CVPR04), I:488-495, 2004.

[24] H. Uemura, S. Ishikawa, and K. Mikolajczyk. "Feature Tracking and Motion Compensation for Action Recognition". In Proc. of BMVA British Machine Vision Conference (BMVC'08), 2008.

[25] P. Viola and M. Jones. "Rapid Object Detection using a Boosted Cascade of Simple Features". In Proc. of IEEE International Conference on Computer Vision and Pattern Recognition (CVPR'01), I:511-518, 2001.

[26] J. Willamowski, D. Arregui, G. Csurka, C. R. Dance, and L. Fan. "Categorizing Nine Visual Classes using Local Appearance Descriptors". In Proc. of IWLAVS, 2004.

[27] G. Willems, T. Tuytelaars, and L. Gool. An Efficient Dense and Scale-Invariant Spatio-Temporal Interest Point Detector. In Proc. of European Conference on Computer Vision (ECCV'08), II:650-663.

[28] S. F. Wong and R. Cipolla. "Extracting Spatio Temporal Interest Points using Global Information". In Proc. of IEEE Int. Conf. on Comp. Vis. (ICCV'07), 2007. 